\title{
Muon identification and performance in the ATLAS experiment
}

\author{
Sébastien Rettie*t \\ on behalf of the ATLAS Collaboration \\ The University of British Columbia - TRIUMF \\ E-mail: sebastien.rettie@cern.ch
}

\begin{abstract}
Muon reconstruction and identification play a fundamental role in many analyses of central importance in the LHC run-2 Physics programme. The algorithms and the criteria used in ATLAS for the reconstruction and identification of muons with transverse momentum from a few $\mathrm{GeV}$ to the $\mathrm{TeV}$ scale will be presented. Their performance is measured in data based on the decays of $\mathrm{Z}$ and $\mathrm{J} / \psi$ to a pair of muons, that provide a large statistics calibration sample. Reconstruction and identification efficiencies are evaluated, as well as momentum scales and resolutions, and the results are used to derive precise MC simulation corrections. Isolation selection criteria and their performances in presence of high pileup will also be presented.
\end{abstract}

XXVI International Workshop on Deep-Inelastic Scattering and Related Subjects (DIS2018)

16-20 April 2018

Kobe, Japan

\footnotetext{
* Speaker.

${ }^{\dagger}$ The author acknowledges support from the Vanier Canada Graduate Scholarship program, and the Natural Sciences and Engineering Research Council of Canada.
} 


\section{Introduction}

Muon reconstruction and identification performance is extremely important in ATLAS both for precision measurements and new physics searches. This note describes the performance of the ATLAS detector [1] at the LHC [2] with respect to muon identification and reconstruction, and outlines the muon reconstruction and isolation efficiencies. The dependence on pileup of various efficiencies is assessed, and the corrections applied to simulation are described. Most of the results presented are based on the work described in [3].

\section{Muon Reconstruction \& Identification}

A full description of the ATLAS detector can be found in [1]. When a muon candidate is reconstructed, a set of five parameters is obtained from the track fit: $\mathrm{M}=\left(\mathrm{d}_{0}, \mathrm{z}_{0}, \phi, \theta, q / p\right)$. A muon traversing the ATLAS detector initially passes through the Inner Detector (ID) and bends in the $\phi$ direction due to a $2 \mathrm{~T}$ solenoid magnetic field. The ID track has very precise hits close to the Interaction Point (IP), which helps to constrain the impact parameters $\mathrm{d}_{0}$ and $\mathrm{z}_{0}$, as well as the angles $\phi$ and $\theta$. After passing through the electromagnetic and hadronic calorimeters, the muon enters the Muon Spectrometer (MS) and bends along the $\theta$ direction due to a toroidal magnetic field. The MS track has better $q / p$ resolution due to its longer lever arm. There are four muon reconstruction algorithms available within the ATLAS detector:

- Combined (CB) muons are obtained by performing a global refit of the ID and MS tracks

- Segment-tagged (ST) muons consist of a fitted ID track and a MS segment

- Calorimeter-tagged (CT) muons consist of a fitted ID track and an energy deposit in the calorimeters

- Extrapolated (ME) muons consist of only a MS track

Figure 1 summarizes the four reconstruction algorithms described above.

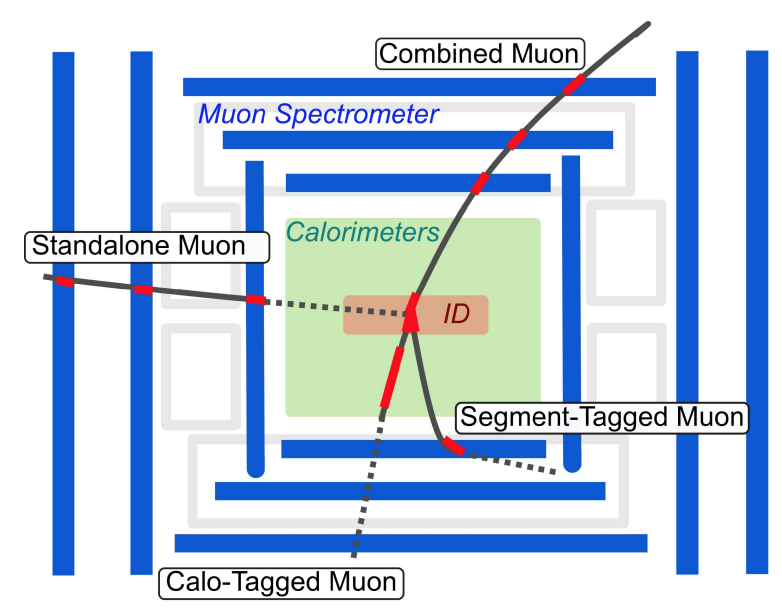

Figure 1: Reconstruction algorithms available in the ATLAS detector. 
Collections of selection criteria, so-called working points (WPs), are defined in order to accommodate the various needs of ATLAS physics analysis groups. A set of five WPs are currently supported. The Loose WP maximizes reconstruction efficiency and uses all types of muons. The Medium WP is used as the default selection for ATLAS and uses only CB and ME muons. The Tight WP maximizes selection purity and uses only CB and ME muons. The Low- $p_{T}$ WP maximizes selection efficiency and fake-rejection for muons having $p_{\mathrm{T}}<5 \mathrm{GeV}$ and is used e.g. in SUSY searches. The High- $p_{T}$ WP maximizes momentum resolution for $p_{\mathrm{T}}>100 \mathrm{GeV}$ and is used e.g. in the $Z^{\prime} \rightarrow \mu \mu$ analysis [4].

\section{Reconstruction Efficiency}

The reconstruction efficiency of muons in ATLAS is estimated using a so-called Tag \& Probe method that uses high-statistics samples of $Z \rightarrow \mu^{+} \mu^{-}$and $J / \psi \rightarrow \mu^{+} \mu^{-}$resonances. The tag is a Medium quality muon that fires the trigger, and the probe is reconstructed by a system independent of the one being studied (e.g. the calorimeter). The reconstruction efficiency is then defined by equation 3.1, which is explained in detail in [3].

$$
\varepsilon(\mathrm{X})=\varepsilon(\mathrm{X} \mid \mathrm{ID}) \cdot \varepsilon(\mathrm{ID})=\varepsilon(\mathrm{X} \mid \mathrm{CT}) \cdot \varepsilon(\mathrm{ID} \mid \mathrm{MS}),\left(\mathrm{X}=\text { Medium } / \text { Tight } / \text { High }-\mathrm{p}_{\mathrm{T}}\right)
$$

Table 1 shows the selection efficiencies for various selection WPs. As seen in figure 2(a), which shows the reconstruction efficiencies as a function of $p_{\mathrm{T}}$, the reconstruction efficiency for Medium muons is $\sim 99 \%$ for $p_{\mathrm{T}}>10 \mathrm{GeV}$ and $|\eta|>0.1$. Figure 2(b) shows the reconstruction efficiency for Medium muons having $|\eta|>0.1$ is $>98 \%$, and that Loose muons recover efficiency for $|\eta|<0.1$.

\begin{tabular}{|c||c|c||c|c|}
\hline \hline \multicolumn{1}{|c||}{} & \multicolumn{2}{c||}{$4<p_{\mathrm{T}}<20 \mathrm{GeV}$} & \multicolumn{2}{c|}{$20<p_{\mathrm{T}}<100 \mathrm{GeV}$} \\
\hline Selection & $\varepsilon_{\mu}^{\mathrm{MC}[\%]}$ & $\varepsilon_{\text {Hadrons }}^{\mathrm{MC}}[\%]$ & $\varepsilon_{\mu}^{\mathrm{MC}}[\%]$ & $\varepsilon_{\text {Hadrons }}^{\mathrm{MC}}[\%]$ \\
\hline Loose & 96.7 & 0.53 & 98.1 & 0.76 \\
Medium & 95.5 & 0.38 & 96.1 & 0.17 \\
Tight & 89.9 & 0.19 & 91.8 & 0.11 \\
High-pT & 78.1 & 0.26 & 80.4 & 0.13 \\
\hline \hline
\end{tabular}

Table 1: Selection efficiencies of various muon selection working points. Table taken from [3].

\section{Isolation Efficiency}

Muon isolation is used for background rejection to reduce the background contributions from light and heavy hadron decays inside jets. Isolation is accomplished by measuring detector activity around a muon candidate. Track-based and calorimeter-based variables are used to quantify isolation. An example of such a variable is shown in figure 3(a). A total of seven isolation selection criteria are optimized for different physics analyses. An example of the efficiency of such a selection, the FixedCutLoose selection, as a function of $p_{\mathrm{T}}$ is shown in figure 3(b). Overall good agreement between data and simulation is observed. 


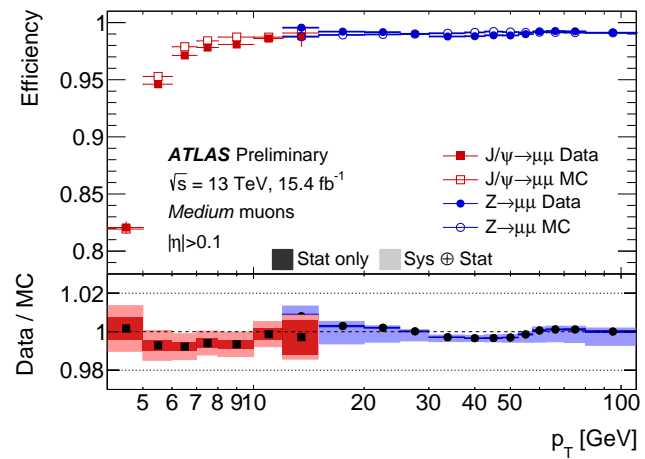

(a)

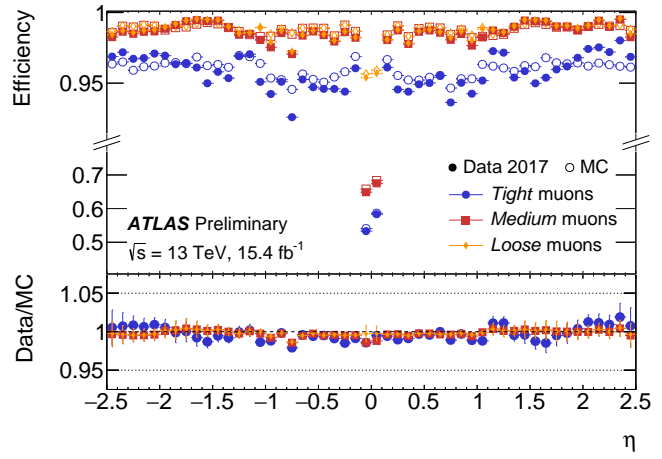

(b)

Figure 2: Reconstruction efficiency as a function of $p_{\mathrm{T}}(2(\mathrm{a}))$ and $\eta(2(\mathrm{~b}))$. Figures taken from [5].

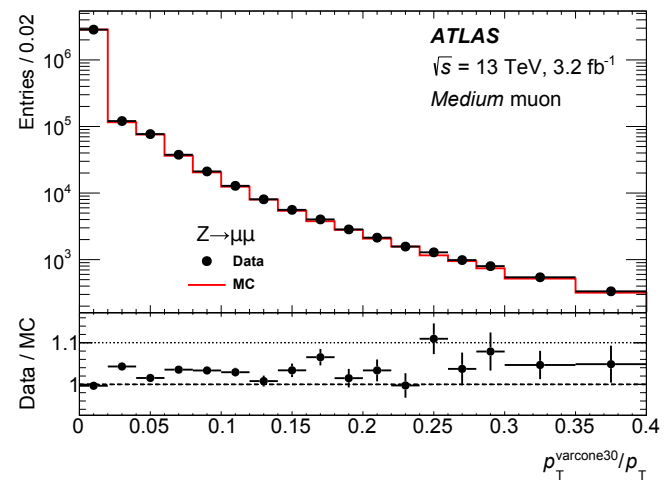

(a)

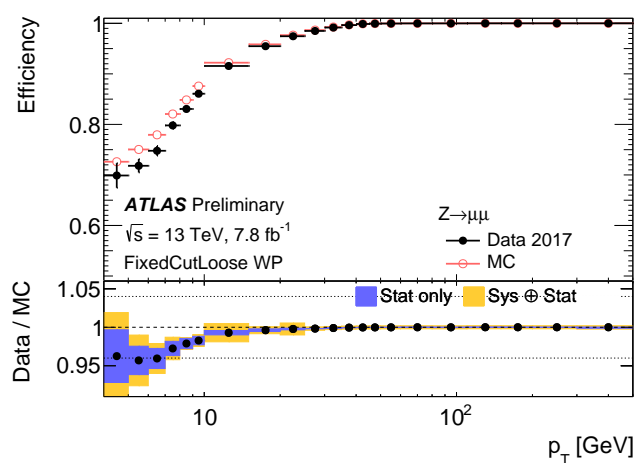

(b)

Figure 3: Track-based isolation variable used for muon isolation (3(a)), and isolation efficiency as a function of $p_{\mathrm{T}}$ for the FixedCutLoose isolation working point (3(b)). Figures taken from [3] and [5].

\section{Pileup Dependance}

The average number of interactions per bunch crossing, or pileup $\langle\mu\rangle$, is continually increasing due to the excellent performance of the LHC. It is thus crucial to study how the various efficiencies discussed above depend on $\langle\mu\rangle$. Figure 4(a) shows a slight loss of efficiency with higher $<\mu>$ with the current isolation working points. No clear dependence of reconstruction efficiency on pileup is observed, as seen in figure 4(b). Performance groups are continually working to improve isolation definitions to be pileup robust; this work is paving the way for the High-Luminosity LHC, where the expected $\langle\mu>$ is $\sim 200$.

\section{Momentum Scale \& Resolution Corrections}

Ideally, the $p_{\mathrm{T}}$ reconstructed in the ID and MS in simulation match exactly the measurements in data. In order to achieve this, corrections are applied to the simulation. Relevant parameters are extracted from data using a binned maximum-likelihood fit with templates derived from simulation. The fit compares the invariant mass of a dimuon system from a $\mathrm{Z}$ or $\mathrm{J} / \psi$ decay and the normalized 


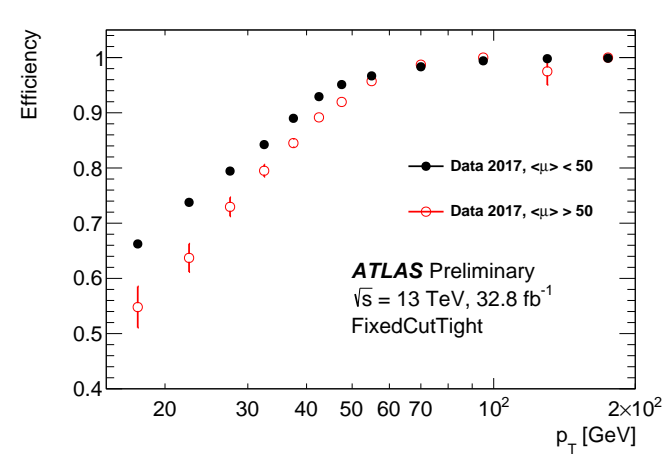

(a)

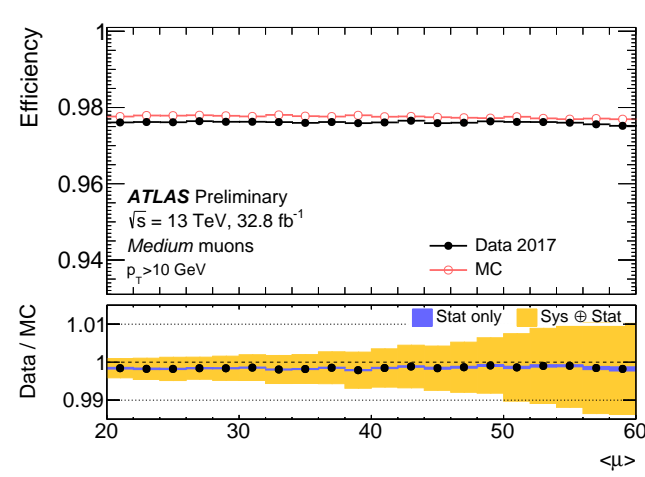

(b)

Figure 4: Isolation efficiency as a function of $p_{\mathrm{T}}$, comparing low and high pileup values for the FixedCutTight (4(a)) WP, and reconstruction efficiency as a function of pileup (4(b)). Figures taken from [6].

ID-MS $p_{\mathrm{T}}$ difference distributions in data and simulation. The ID and the MS are calibrated separately. After applying the corrections, the line shape of the $\mathrm{Z}$ peak between data and simulation match very well within systematic uncertainty, as seen in figure 5 .

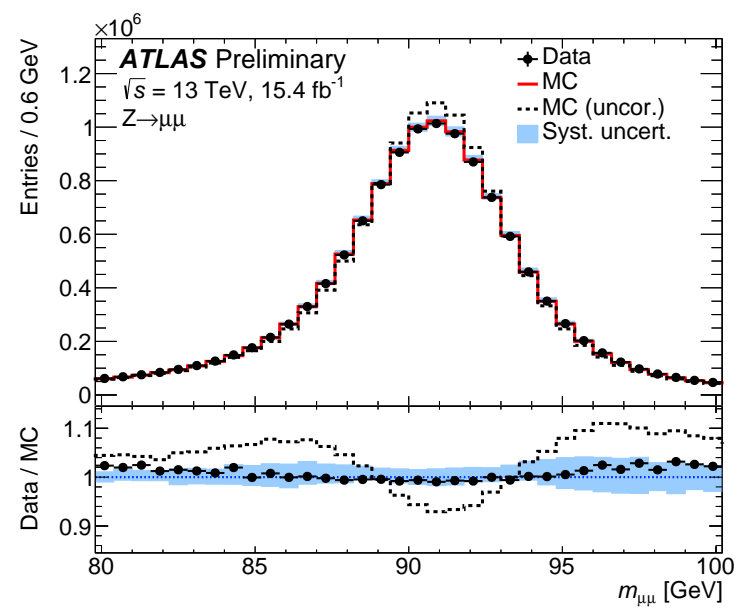

Figure 5: Effect of applying momentum corrections to simulation. Figure taken from [5].

The scale and resolution of the dimuon invariant mass are then measured through a Crystal Ball function; the tail of the Crystall Ball function models the energy loss of the muons. As seen in figure 6 , the scale agreement between data and simulation is at the per mille level, and the resolution agreement is at the percent level.

\section{Future Work}

ATLAS is continuously improving its reconstruction software. An example of this is the implementation of a new type of object: Alignment Effects On Tracks (AEOTs). In the previous versions of the ATLAS reconstruction software, the final track errors were inflated to account for 


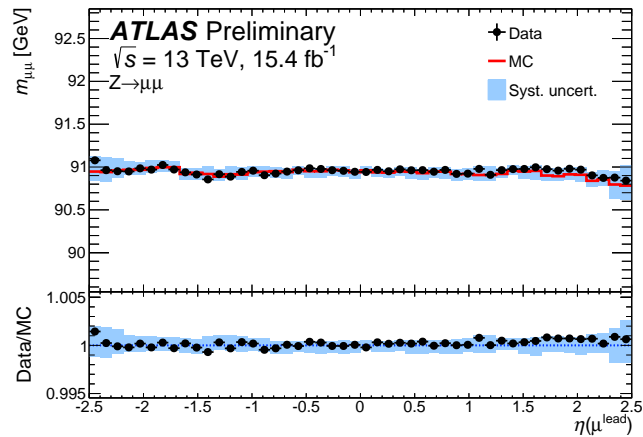

(a)

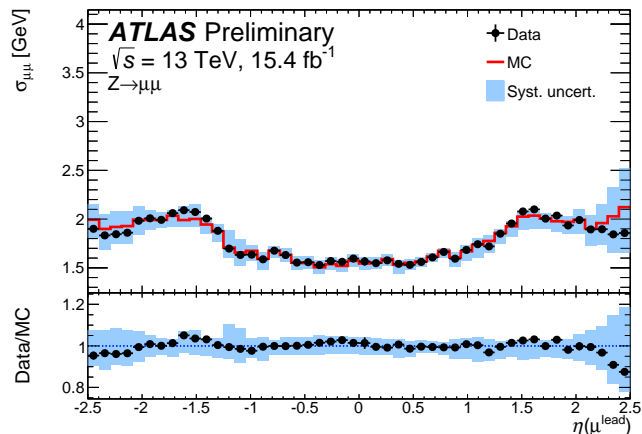

(b)

Figure 6: Momentum scale (6(a)) and resolution (6(b)). Figures taken from [5].

alignment uncertainties; this was equivalent to simply deweighting certain chambers in the global refit. This was done only in specific critical situations such as Barrel/Endcap overlap regions or Small/Large sector overlap regions. In the new version of the software, alignment discontinuities are now fit. This allows for a more realistic error estimate on the $q / p$ measurement, and might allow recovery of previously vetoed MS regions for High- $p_{T}$ muons. This is made possible by AEOTs, which specify position and angle uncertainties on chamber hits. The combined track fit is thus performed using Gaussian constraints on chamber hits where alignment uncertainties are used as the Gaussian widths. This concept is pictorially described in figure 7.

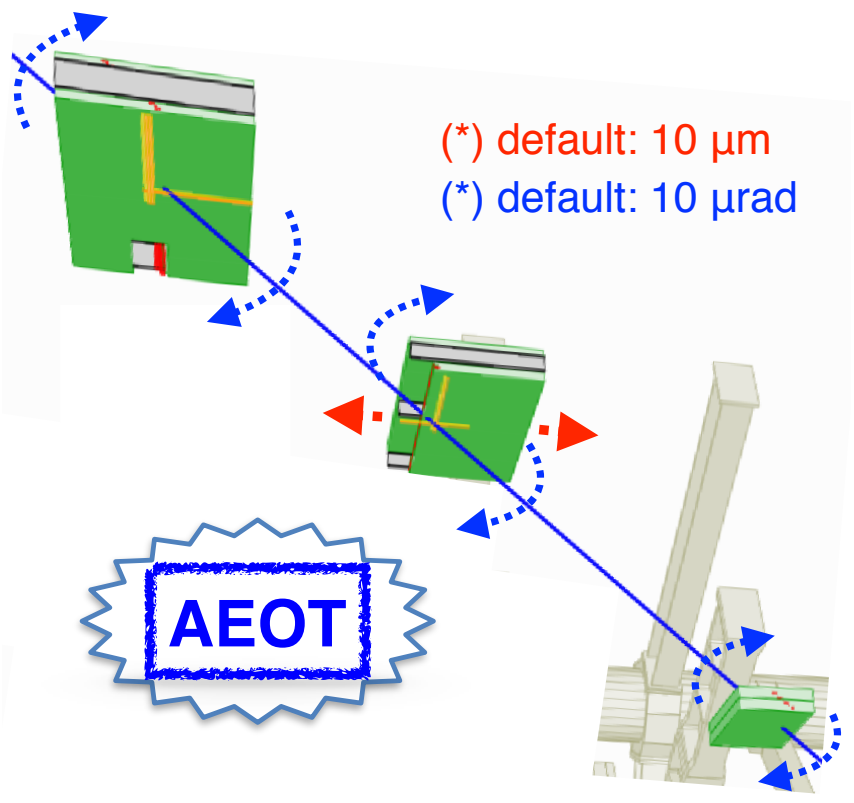

Figure 7: Visual representation of Alignment Effects On Track (AEOT).

\section{Conclusion}

The muon reconstruction and identification performance of the ATLAS detector at the LHC 
was measured using data based on the decays of $\mathrm{Z}$ and $\mathrm{J} / \psi$ resonances to a pair of muons taken during the 2015-2017 Run 2 data-taking period. The detector reconstruction efficiency is $\sim 99 \%$ for Medium muons with $p_{\mathrm{T}}>10 \mathrm{GeV}$. In addition, isolation selection criteria and their performances were evaluated. In presence of high pileup, the reconstruction efficiency remains constant. The agreement between data and simulation was shown to be at the per mille level for the dimuon invariant mass scale, and at the percent level for the dimuon invariant mass resolution. Finally, the ATLAS reconstruction software is continuously improving, e.g. the implementation of AEOTs provides a more realistic description of the $q / p$ measurement uncertainty.

\section{References}

[1] ATLAS Collaboration, The ATLAS Experiment at the CERN Large Hadron Collider, JINST 3 (2008) S08003

[2] L. Evans and P. Bryant (editors), The CERN Large Hadron Collider: Accelerator and Experiments, JINST 3 (2008) S08001

[3] ATLAS Collaboration, Muon reconstruction performance of the ATLAS detector in proton-proton collision data at $\sqrt{s}=13$ TeV, Eur. Phy. J. C 76 (2016) 292

[4] ATLAS Collaboration, Search for new high-mass phenomena in the dilepton final state using $36 \mathrm{fb}^{-1}$ of proton-proton collision data at $\sqrt{s}=13$ TeV with the ATLAS detector, J. High Energ. Phys. 10 (2017) 182

[5] ATLAS Collaboration, https://atlas.web.cern.ch/Atlas/GROUPS/PHYSICS/PLOTS/MUON-2017-002/index.html

[6] ATLAS Collaboration, https://atlas.web.cern.ch/Atlas/GROUPS/PHYSICS/PLOTS/MUON-2017-005/index.html 